\title{
Based on the KTP crystal double frequency laser experiment system
}

\section{research}

\author{
Feng Hui Cao ${ }^{1, a}$,Yue $\mathrm{li}^{1, \mathrm{~b}}$, Yun Feng Dong ${ }^{1, \mathrm{c}}$, Yu Ling Wang ${ }^{1, \mathrm{~d}}$ and Xin Chen ${ }^{1, \mathrm{e}}$ \\ ${ }^{1}$ School Of Mechatronic Engineering, Daqing Normal University, Daqing 163712, China \\ acaofenghui@126.com, ${ }^{\mathrm{b}} 5500752 @ q q . c o m,{ }^{\mathrm{b}} 5500752 @ q q . c o m,{ }^{\mathrm{c}} 694846035 @ q q . c o m,{ }^{\mathrm{d}} 67503601 @$ \\ qq.com, ${ }^{\mathrm{e}} 45340424 @ q q . c o m$.
}

Keywords: laser; Frequency multiplication technology ;KTP crystal

\begin{abstract}
At first, this paper has summarized the theory of frequency doubling crystal, summarized suitable for uv frequency doubling of the characteristics of nonlinear optical crystal, put forward the theory basis of frequency doubling crystal KTP crystal, debug out of efficient cavity frequency doubling green laser, frequency conversion of $49.3 \%$.
\end{abstract}

\section{Introduction}

Nonlinear optical frequency conversion technology has been a hot research topic in the field of laser technology. Because it can work without using the new laser material under the condition of extension laser wavelengths, open up new application fields. Today can be a variety of frequency is obtained by frequency conversion device of high power laser, also can from the infrared to the ultraviolet wavelength range. The invention of the latest high quality nonlinear optical crystals, nonlinear optical frequency conversion technology is widely used, but also make a branch of the door has become the most active in nonlinear optical fields, one of the most mature branch.

\section{Summary of frequency multiplication principle}

Since the laser appears, it is enough to reflect light material nonlinear response of the light field. This related to the intensity of optical effect, often referred to as nonlinear optical effect. In only as a description of it, will be launched for the nonlinear optical medium CLP polarization P outside light field $\mathrm{E}$ form of power series, namely

$$
\vec{P}=\varepsilon_{0}\left[\chi^{(1)} \vec{E}+\chi^{(2)} \vec{E} \vec{E}+\chi^{(3)} \vec{E} \vec{E} \vec{E}+\cdots \cdots\right]
$$

Consider medium under quadratic nonlinear electric polarization effect, if there are two columns of different frequency of plane wave in the crystal reached the match in place, set for the $\mathrm{Z}$ axis direction,

$$
\begin{aligned}
& \mathrm{E}_{1}(\mathrm{Z}, \mathrm{T})=\mathrm{E}_{1} \cos \left(\omega_{1} \mathrm{t}-\mathrm{k}_{1} \mathrm{Z}\right) \\
& \mathrm{E}_{2}(\mathrm{Z}, \mathrm{T})=\mathrm{E}_{2} \cos \left(\omega_{2} \mathrm{t}-\mathrm{k}_{2} \mathrm{Z}\right) \\
& \mathrm{P}=\varepsilon_{0} \chi^{(1)}\left[\mathrm{E}_{1}(\mathrm{Z}, \mathrm{T})+\mathrm{E}_{2}(\mathrm{Z}, \mathrm{T})\right]+\varepsilon_{0} \chi^{(2)}\left[\mathrm{E}_{1}(\mathrm{Z}, \mathrm{T})+\mathrm{E}_{2}(\mathrm{Z}, \mathrm{T})\right]^{2}
\end{aligned}
$$

Polarized wave not only contains the fundamental frequency $\omega_{1} 、 \omega_{2}$, omega, omega also appear frequency doubling, and frequency and difference frequency, obviously if the incident two columns 
of wave frequency for $\omega_{1}$ and $2 \omega_{1}$ omega, omega and the frequency of omega $3 \omega_{1}$, that is three times the frequency. By the same frequency multiplication technology can converts the frequency of omega $\omega_{1}$ laser frequency to $2 \omega_{1}$ green, omega travel through the frequency doubling frequency can be obtained as 4 omega $4 \omega_{1}$ deep ultraviolet laser.

\subsection{Phase matching principle}

Phase matching is a very important concept in the nonlinear optics. Phase matching technology adopted, the nonlinear effect of conversion efficiency greatly improved[1]. Light travels in a nonlinear crystal in the phase velocity depends on the light on the direction of propagation of refractive index, and it is associated with the polarization and the direction of propagation of light waves. Direction of propagation is determined, the general said, there are two to determine the characteristics of the phase velocity and the polarization direction of eigen waves, and their polarization direction perpendicular to each other, if the polarization direction of a light and direction of these two characteristics of a parallel, then the light through the crystal, its polarization direction will remain unchanged, analysis of light wave propagation in nonlinear crystals, is to solve the two characteristics of the waves of light polarization direction and the corresponding phase velocity (or refractive index). And use various methods to control crystal, its purpose is to change the refractive index, the fundamental frequency and frequency doubling light refractive index of the surface tangent in perpendicular to the axis of the plane, also is the fundamental frequency light refractive index and the frequency doubling light refractive index is equal. Without considering the crystal light absorption and scattering conditions, from the fundamental frequency to frequency doubling light a light quantum system comply with the energy and momentum conservation.

\subsection{Biaxial crystals in three waves interaction phase matching}

According to the polarization direction of incident base wave, phase matching method of can be divided into two categories: the first class I and class II. Such as the incident wave are slow light, polarization direction parallel, called type I phase matching; Such as incident wave both slow and fast light, orthogonal polarization direction, it is called a type II phase matching. The same as the uniaxial crystal, phase matching problem, can turn ratio for the refractive index of the intersecting line problem. As a result of the fast and slow light of biaxial crystal refractive index, respectively for the refractive index of the inner shell surface and the outer shell surface, so I kind of match is composed of fundamental wave slow light and fast light refractive index of the space of the harmonic line, namely by the fundamental harmonic of inner shell surface and exempt from the line[4]. Class II phase matching is fundamental wave refraction surface inside and outside layer on the surface of the shell and the average shell surface and the harmonic on the surface of the inner space. The phase matching condition of electric field can be expressed as:

$$
\begin{array}{ll}
\text { I } & \vec{E}\left(\omega 1, n_{1}^{\prime}\right)+\vec{E}\left(\omega 2, n_{2}^{\prime}\right) \rightarrow \vec{E}\left(\omega 3, n_{3}{ }^{\prime \prime}\right) \\
\text { II } \quad \vec{E}\left(\omega 1, n_{1}^{\prime}\right)+\vec{E}\left(\omega 2, n_{2}^{\prime \prime}\right) \rightarrow \vec{E}\left(\omega 3, n_{3}{ }^{\prime \prime}\right)
\end{array}
$$

\section{The nonlinear characteristics of frequency doubling crystal}

Choose the basis of nonlinear crystal is should have a larger effective nonlinear coefficient, within the scope of the wide spectrum of high transparency, as far as possible in need of band phase matching, easy to implement with strong light damage resistance and easy for realization of crystal growth and processing.

Oxygen titanium potassium phosphate crystals $\left(\mathrm{KTiOPO}_{4}\right) \mathrm{KTP}$ crystals, is negative biaxial crystal, the 
lattice structure of orthogonal crystal system, density $2.945 \mathrm{~g} / \mathrm{cm}^{3}$, pervious to light band is 350-4500nm,

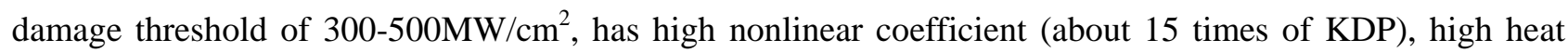
conductivity (2 times of BNN crystals), moisture absorption and deliquescence, without separation under $900^{\circ} \mathrm{C}$, good mechanical properties and crystal surface polishing, easily mismatch degree is small.

KTP crystal when he worked in short wavelength absorption coefficient is greater than the long wavelength, so when running under the condition of short wavelength, pay attention to the heat dissipation of KTP crystal, in order to avoid thermal damage. KTP crystal frequency doubling of the shortest wavelength of $994 \mathrm{~nm}$, harmonic is $497 \mathrm{~nm}$, wavelength less than $994 \mathrm{~nm}$, matching points, there is no class II and class I matching the nonlinear coefficient is far less than class II, so I basic don't match.

Because the KTP crystal has high nonlinear coefficient, pervious to light band width, by widespread application Yu Beipin, and frequency, and in the process of optical parametric, especially suitable for $\mathrm{Nd}^{3+}$ :YAG laser (wavelength 1064nm) as The Times of experiments.

Three lithium borate crystals $\left(\mathrm{LiB}_{3} \mathrm{O}_{5}\right)$ LBO crystal [4], is a good high-power ultraviolet frequency doubling crystal, have wide at the same time allow Angle and small off the Angle, has been successfully used in YAG, YLF and YAP laser double and triple frequency, belong to the negative biaxial crystal, the crystal structure of orthorhombic system, melting point at $834^{\circ} \mathrm{C}$, density $2.47 \mathrm{~g} / \mathrm{cm}^{3}$, pervious to light with wide band, pervious to light cover a wide range of $160-2600 \mathrm{~nm}$, effective nonlinear coefficient (SHG) which is about three times that of KDP. Three lithium borate crystal has a wide scope of class I and class II non-critical phase matching, so the critical phase matching can be adopted to overcome the walked away from the effect to obtain high frequency doubling efficiency, and at the same time by the dispersion equation, we can calculate its temperature matching features.

BBO crystal ( $\left.\beta-\mathrm{BaB}_{2} \mathrm{O}_{4}\right)$,is negative uniaxial crystals, is so far can be used in ultraviolet band is one of the most excellent nonlinear crystals. It is widely used for $\mathrm{Nd}^{3+}: \mathrm{YAG}, \mathrm{Nd}^{3+}: \mathrm{YVO}_{4}$ laser second, three, four and five harmonics, high efficiency have been obtained respectively 532nm, 355nm to $266 \mathrm{~nm}$ and $213 \mathrm{~nm}$ laser output. BBO crystal with wide light wavelengths $(189 \mathrm{~nm}$ to $3500 \mathrm{~nm})$, over a wide range of wavelengths can realize class I and class II phase matching (410nm to 3500nm), high frequency doubling conversion efficiency (the equivalent of six times that of the KDP crystal), optical uniformity, good temperature receiving wide Angle $\left(55^{\circ} \mathrm{C}\right.$ or so).

Below is a list of KTP、LBOand BBOcrystal damage threshold comparison, conditions for

Table 1 two crystal damage threshold

\begin{tabular}{l|ll|l|l}
\hline $\begin{array}{l}\text { Crystal } \\
\text { type }\end{array}$ & $\begin{array}{l}\text { Energy density } \\
(\mathrm{J} / \mathrm{cm} 2)\end{array}$ & $\begin{array}{l}\text { Power density } \\
(\mathrm{GW} / \mathrm{cm} 2)\end{array}$ & Ratio \\
KTP & 6.0 & 4.6 & 1 \\
LBO & 24.6 & 18.9 & 4.10 \\
BBO & 12.9 & 9.9 & 2.15 \\
\hline
\end{tabular}

\section{Experimental apparatus and conclusions}

We have adopted the 808 - nm LD as a pump sources are used, and provide focus lens focus after irradiation in the laser crystal neodymium-doped yttrium vanadate $\left(\mathrm{Nd}^{3+}: \mathrm{YVO}_{4}\right)$, through the concave mirror (output mirror) laser resonant cavity, resulting in a 1064nm pulse output, cavity using KTP crystal frequency doubling, because of the type I phase matching nonlinear coefficient is very small, generally do not use, so 
we adopt class II phase matching. For type II phase matching requires fundamental frequency light polarization direction orthogonal to each other, thus, when two mutually orthogonal component numerically equal to the fundamental wave, within the crystal frequency doubling efficiency is highest. Therefore, experiment, KTP crystal $45^{\circ}$ Angle placed, to achieve maximum efficiency of frequency doubling. In the experiment KTP crystal transformation frequency of $49.3 \%$.

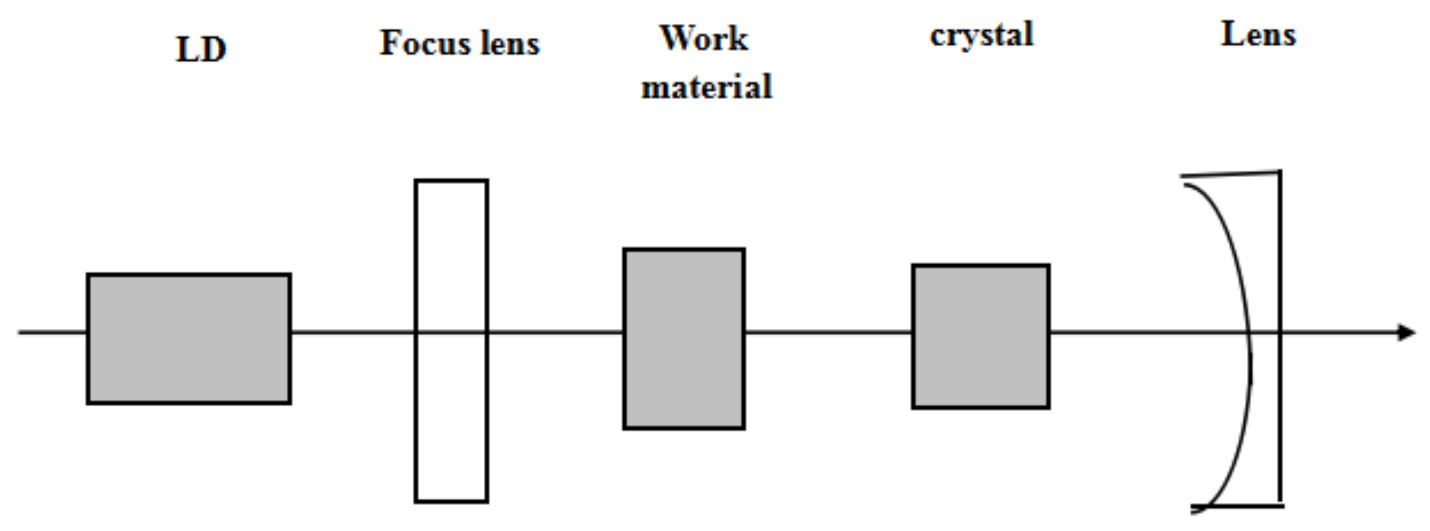

Figure 1 experiment device structure diagram

\section{Conclusion}

This paper first describes the theory of frequency doubling crystal, summarized suitable for double frequency characteristics of the nonlinear optical crystals, put forward the theory of frequency doubling crystal choice, choose KTP crystal design Nd3 + : YAG green laser, the stability of $532 \mathrm{~nm}$ laser output, the conversion efficiency of $49.3 \%$.

\section{Acknowledgements}

This work is daqing guidance of science and technology plan projects (zd-2016-015)

\section{References}

[1] Xu Jianjun, Xu Yan-chao, Yan, Li-me,et.al. Research on the method of optimal PMU placement. International Journal of Online Engineering,v9, S7, p24-29, 2013

[2] Xu Jian-Jun, Y. Y. Zi., Numerical Modeling for Enhancement of Oil Recovery via Direct Current. International Journal of Applied Mathematics and Statistics，2013，43（13）: 318-326

[3] Longchao, Zhu Jianjun, Xu; Limei, Yan. Research on congestion elimination method of circuit overload and transmission congestion in the internet of things. Multimedia Tools and Applications, p 1-20, June 27, 2016

[4] Yan Limei, Zhu Yusong, Xu Jianjun,et.al. Transmission Lines Modeling Method Based on Fractional Order Calculus Theory. TRANSACTIONS OF CHINA ELECTROTECHNICAL SOCIETY, 2014 ,Vol.29,No. 9:260-268 (In Chinese)

[5] YAN Li-mei, CUI Jia, XU Jian-jun,et.al. Power system state estimation of quadrature Kalman filter based on PMU/SCADA measurements. Electric Machines and Control. 2014, Vol.18 
No.6,: 78-84. (In Chinese)

[6] YAN Limei,XIE Yibing, XU Jianjun, et.al. Improved Forward and Backward Substitution in Calculation of Power Distribution Network with Distributed Generation. JOURNAL OF XI'AN JIAOTONG UNIVERSITY,2013, Vol.47, No.6, p117-123. (In Chinese)

[7] Xu J.J., Gai D., Yan L.M. A NEW FAULT IDENTIFICATION AND DIAGNOSIS ON PUMP VALVES OF MEDICAL RECIPROCATING PUMPS. Basic \& Clinical Pharmacology \& Toxicology, 2016,118 (Suppl. 1), 38-38 